\title{
TU/e EmonONEN

\section{Generalized Hopf fibration and geometric SO(3) reduction of the 4DOF harmonic oscillator}

\section{Citation for published version (APA):}

Meer, van der, J. C., Crespo, F., \& Ferrer, S. (2014). Generalized Hopf fibration and geometric SO(3) reduction of the 4DOF harmonic oscillator. (CASA-report; Vol. 1413). Technische Universiteit Eindhoven.

Document status and date:

Published: 01/01/2014

\section{Document Version:}

Publisher's PDF, also known as Version of Record (includes final page, issue and volume numbers)

\section{Please check the document version of this publication:}

- A submitted manuscript is the version of the article upon submission and before peer-review. There can be important differences between the submitted version and the official published version of record. People interested in the research are advised to contact the author for the final version of the publication, or visit the $\mathrm{DOI}$ to the publisher's website.

- The final author version and the galley proof are versions of the publication after peer review.

- The final published version features the final layout of the paper including the volume, issue and page numbers.

Link to publication

\section{General rights}

Copyright and moral rights for the publications made accessible in the public portal are retained by the authors and/or other copyright owners and it is a condition of accessing publications that users recognise and abide by the legal requirements associated with these rights.

- Users may download and print one copy of any publication from the public portal for the purpose of private study or research.

- You may not further distribute the material or use it for any profit-making activity or commercial gain

- You may freely distribute the URL identifying the publication in the public portal.

If the publication is distributed under the terms of Article 25fa of the Dutch Copyright Act, indicated by the "Taverne" license above, please follow below link for the End User Agreement:

www.tue.nl/taverne

Take down policy

If you believe that this document breaches copyright please contact us at:

openaccess@tue.nl

providing details and we will investigate your claim. 


\section{EINDHOVEN UNIVERSITY OF TECHNOLOGY}

Department of Mathematics and Computer Science

\section{CASA-Report I4-I3 \\ April 20I4}

Generalized Hopf fibration and geometric $\mathrm{SO}(3)$ reduction of the 4 DOF harmonic oscillator

by

J.C. van der Meer, F. Crespo, S. Ferrer

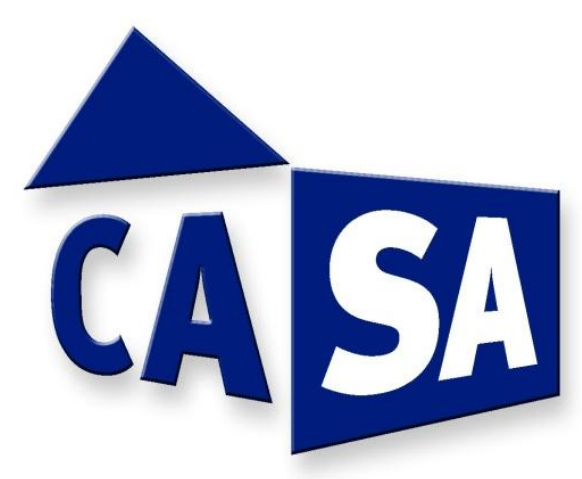

Centre for Analysis, Scientific computing and Applications

Department of Mathematics and Computer Science

Eindhoven University of Technology

P.O. Box $5 \mathrm{I} 3$

5600 MB Eindhoven, The Netherlands

ISSN: 0926-4507 


\title{
Generalized Hopf fibration and geometric $\mathrm{SO}(3)$ reduction of the $4 \mathrm{DOF}$ harmonic oscillator
}

\author{
J.C. van der Meer \\ Faculteit Wiskunde en Informatica, Technische Universiteit Eindhoven, The Netherlands. \\ F. Crespo, S. Ferrer \\ Departamento de Matemática Aplicada, Universidad de Murcia, Spain.
}

\begin{abstract}
It is shown that the generalized Hopf map $\mathbb{H} \times \mathbb{H} \rightarrow \mathbb{H} \times \mathbb{R} \times \mathbb{R}$ in quaternion formulation can be interpreted as an $S O(3)$ orbit map for a symplectic $S O(3)$ action. As a consequence the generalized Hopf fibration $\mathbb{S}^{7} \rightarrow \mathbb{S}^{4}$ appears in the $S O(3)$ geometric symplectic reduction of the $4 \mathrm{DOF}$ isotropic harmonic oscillator.
\end{abstract}

Key Words: Hopf map, Hopf fibration, symplectic reduction, harmonic oscillator.

AMS Subject Classification: primary: 53D20, 37J15, secondary: 55R10.

\section{Introduction}

It is well known that one may reduce the phase space of the 2DOF harmonic oscillator by using the symmetry given by the $\mathbb{S}^{1}$-action generated by the flow of the Hamiltonian. Restricting the reduction mapping to the three spheres given by the energy surfaces, the orbit mapping becomes a reduction of the energy surface $\mathbb{S}^{3}$ to the reduced phase space $\mathbb{S}^{2}$, which mapping is exactly the Hopf fibration $\mathbb{S}^{3} \rightarrow \mathbb{S}^{2}$. See [3]. In section 2. we will make this precise starting from the classical description of the Hopf fibration in complex coordinates. In the following sections we generalize these ideas to the 4DOF isotropic harmonic oscillator. For this purpose we will introduce quaternions in section 3 . and the generalized generalized Hopf map in terms of quaternions in section 4 . In section 5 we will show that, after choosing an appropriate real representation, this Hopf map can be considered as an orbit map for an appropriately chosen symplectic $S O(3)$ action, and that restriction to an energy surface for the harmonic oscillator, gives the generalized Hopf fibration $\mathbb{S}^{7} \rightarrow \mathbb{S}^{4}$. In the final section we will say something about how the twistor fibration, that appears in the diagram for the generalized Hopf fibration, fits in the scheme of symplectic reduction. 


\section{Hopf fibration in $2 \mathrm{DOF}$}

In this section we wil start with the common complex description of the Hopf map and show how this leads to an orbit map and reduction map for a 2DOF harmonic oscillator.

For the ambient space of the classical Hopf fibration, consider the complex vector space $\mathbb{C}^{2}$, together with the usual hermitian inner product. Let $z \in \mathbb{C}^{2}$ and $w \in \mathbb{C}^{2}$, where $z=\left(z_{1}, z_{2}\right), w=\left(w_{1}, w_{2}\right)$ and $z_{1}, z_{2}, w_{1}, w_{2} \in \mathbb{C}$, then

$$
<z, w>=\bar{z} w=\bar{z}_{1} w_{1}+\bar{z}_{2} w_{2} .
$$

Thus $\mathbb{S}^{3}$ can be shown as a subset in $\mathbb{C}^{2}$ given by $\mathbb{S}^{3}=\left\{z \in \mathbb{C}^{2} \mid<z, z>=1\right\}$. Define an equivalence relation on $\mathbb{S}^{3}$ by $z \sim \omega$ iff $\omega=\lambda z, \lambda \in \mathbb{S}^{1}$. We get that

$$
\mathbb{S}^{3} / \sim \cong P\left(\mathbb{C}^{2}\right)=\mathbb{C P}^{1}
$$

Therefore, by means of the stereographic projection $\mathbb{C} P^{1} \rightarrow \mathbb{S}^{2}$, the classic Hopf fibration $\Pi: \mathbb{S}^{3} \rightarrow \mathbb{S}^{2}$ is built in a constructive process that we may describe by means of the commutative diagram in figure (1).

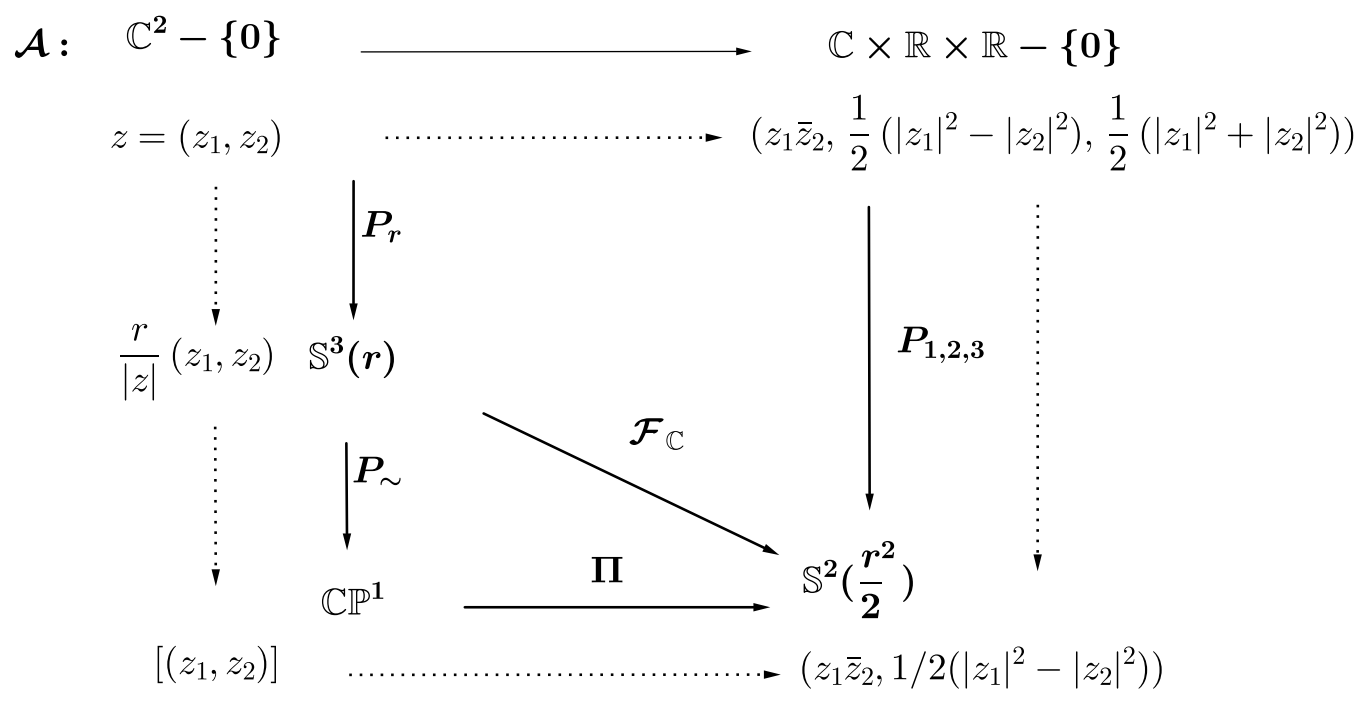

Figure 1: Hopf map and classic Hopf fibration diagram.

Here $\Pi$ is the stereographic projection, $P_{r}$ is the map from $\mathbb{C}^{2}-\{0\}$ to $\mathbb{S}^{3}(r)$ that matches each semi-ray through the origin with the corresponding element of module equal to $r$, $P \sim$ from $\mathbb{S}^{3}(r)$ into $\mathbb{C} P^{1}$ identifies each point in the sphere to its corresponding class of equivalence, $P_{1,2,3}$ is the elimination of the fourth component and $\mathcal{A}$ is the Hopf map. The Hopf fibration $\mathcal{F}_{r}$ from $\mathbb{S}^{3}(r)$ to $\mathbb{S}^{2}\left(\frac{r}{2}\right)$ is given by composing $\Pi$ and $P_{\sim}$, it can also 
be obtained by means of the restriction of $\mathcal{A}$, the Hopf map, to $\mathbb{S}^{3}(r)$ and composing it with $P_{1,2,3}$.

Notice that $\Pi: \mathbb{C} P^{1} \longrightarrow \mathbb{S}^{2}\left(\frac{r}{2}\right)$ is based on the classic stereographic projection from $\mathbb{S}^{2}\left(\frac{r}{2}\right)-N$ onto the real plane, in this case the north pole is covered by the infinite point $[(1,0)]$.

Moving to cartesian coordinates, and taking into account the natural relation between $\mathbb{C}$ and $\mathbb{R}^{2}$, by setting $z=\left(z_{1}, z_{2}\right)=\left(q_{1}+i q_{2}, Q_{1}+i Q_{2}\right)$ we get $\mathcal{A}_{\mathbb{R}}: \mathbb{R}^{4} \rightarrow \mathbb{R}^{4}$ given by

$$
\mathcal{A}_{\mathbb{R}}\left(q_{1}, q_{2}, Q_{1}, Q_{2}\right)=\left(\omega_{1}(q, Q), \omega_{2}(q, Q), \omega_{3}(q, Q), \omega_{4}(q, Q)\right),
$$

where

$$
\begin{aligned}
\omega_{1} & =q_{1} Q_{2}-q_{2} Q_{1}, & \omega_{3} & =\frac{1}{2}\left(q_{1}^{2}+q_{2}^{2}-Q_{1}^{2}-Q_{2}^{2}\right), \\
\omega_{2} & =q_{1} Q_{1}+q_{2} Q_{2}, & \omega_{4} & =\frac{1}{2}\left(q_{1}^{2}+q_{2}^{2}+Q_{1}^{2}+Q_{2}^{2}\right),
\end{aligned}
$$

and the following relation between the $\omega_{i}^{\prime} s$ holds

$$
\mathcal{C}\left(q_{1}, q_{2}, Q_{1}, Q_{2}\right)=\omega_{1}^{2}+\omega_{2}^{2}+\omega_{3}^{2}-\omega_{4}^{2}=0 .
$$

Notice that the image of $\mathbb{C}^{2}-\{0\}$ by the Hopf map is given by the algebraic manifold defined in (1) by $\mathcal{C}$, it is a 3 -dimensional cone with vertex in the coordinate origin. If we consider $\mathbb{R}^{4}$ together with the standard symplectic form then the polynomials $\omega_{i}$ span a Lie algebra with bracket being the Poissonbracket induced by the symplectic form. $\omega_{1}$ is in the center of this Lie algebra, ie. we have $\left\{\omega_{1}, \omega_{i}\right\}=0$ for $i=2,3,4$. Thus this Hopf map is an orbit map for the symplectic $S^{1}$-action generated by the Hamiltonian $\omega_{1}$ with respect to the standard symplectic form on $\mathbb{R}^{4}$. Restriction to $\omega_{4}^{-1}(c)$, i.e. to levelsets of $\omega_{4}$, then gives a Hopf fibration $S^{1} \hookrightarrow S^{3} \rightarrow S^{2}$, where the fibre is the orbit for the $\omega_{1}$-action. Although this restriction of the Hopf map does correspond to a reduction of the manifold $\omega_{4}^{-1}(c)$ for the $\omega_{1}$-action, it is not a reduction with respect to the $\omega_{4}$-action.

However, one can give a construction of the Hopf map such that it will be an orbit map for the $\omega_{4}$-action and such that the reduction map wil be a Hopf fibration with the orbit of the $\omega_{4}$-action as a fibre, as will be shown below.

Consider $\mathbb{R}^{4}$ together with the standard symplectic form. The above given real representation of the Hopf map is not an orbit map for the symplectic action of $\omega_{4}$. To obtain a real representation which is an orbit map consider the real representation obtained by setting $z=\left(z_{1}, z_{2}\right)=\left(q_{1}-i Q_{1}, q_{2}-i Q_{2}\right)$, which gives

$$
\tilde{\mathcal{A}}_{\mathbb{R}}\left(q_{1}, q_{2}, Q_{1}, Q_{2}\right)=\left(\tau_{1}(q, Q), \tau_{2}(q, Q), \tau_{3}(q, Q), \tau_{4}(q, Q)\right),
$$


where

$$
\begin{array}{ll}
\tau_{1}=q_{1} Q_{2}-q_{2} Q_{1}, & \tau_{3}=\frac{1}{2}\left(\left(q_{1}^{2}+Q_{1}^{2}\right)-\left(q_{2}^{2}+Q_{2}^{2}\right)\right), \\
\tau_{2}=q_{1} q_{2}+Q_{1} Q_{2}, & \tau_{4}=\frac{1}{2}\left(q_{1}^{2}+q_{2}^{2}+Q_{1}^{2}+Q_{2}^{2}\right)=\omega_{4},
\end{array}
$$

and the following relation between the $\tau_{i}^{\prime} s$ holds

$$
\mathcal{C}\left(q_{1}, q_{2}, Q_{1}, Q_{2}\right)=\tau_{1}^{2}+\tau_{2}^{2}+\tau_{3}^{2}-\tau_{4}^{2}=0
$$

Then $\tau_{4}$ is the Hamiltonian of the harmonic oscillator. The invariants for the symplectic $\mathbb{S}^{1}$-action generated by the flow of the Hamiltonian vector field corresponding to $\tau_{4}$ with respect to the standard symplectic form are $\tau_{1}, \tau_{2}, \tau_{3}$, and $\tau_{4}$. Consequently we have that $\tilde{\mathcal{A}}_{\mathbb{R}}$ is the orbit map for this $\mathbb{S}^{1}$-action. Restriction of $\mathcal{A}_{\mathbb{R}}$ to level surfaces $\tau_{4}=c$ then gives a map $\mathbb{S}^{3} \rightarrow \mathbb{S}^{2}$, which is a reduction map with the 2 -sphere given by $\tau_{1}^{2}+\tau_{2}^{2}+\tau_{3}^{2}=c^{2}$ as a reduced phase space.

\section{Preliminaries on quaternions}

The generalization of the Hopf map that we give next is based upon the generalization from the complex numbers to the quaternions. For that reason, we provide here a brief description of the division ring $\mathbb{H}$, that also will set up a fixed notation through this note.

The hyper-complex numbers of rank 4 were invented by W. R. Hamilton [11], he gave them the name of quaternion, it is a division ring $\mathbb{H}$, the elements of which are denoted by $\mathbf{q}=\left(q_{1}, q\right) \in \mathbb{H}$, and may be regarded as a real part $q_{1}$ plus the imaginary vector part $q=\left(q_{2}, q_{3}, q_{4}\right) \in \mathbb{R}^{3}$. Quaternions having zero scalar part are called pure quaternions and can regarded both as a vector in $\mathbb{R}^{3}$ and as a quaternion. Therefore there is a bijective identification between $\mathbb{R}^{3}$ and the pure quaternions.

The quaternions, together with the operations of addition $(+)$ by components and scalar multiplication $(\cdot)$, may be identified with $\mathbb{R}^{4}$ as a vector space. The quaternionic multiplication (o) provide a division ring structure to $\mathbb{H}$.

It is customary to use the notation $\{\mathbf{1}, \mathbf{i}, \mathbf{j}, \mathbf{k}\}$ for a basis in $(\mathbb{H},+, \mathbb{H})$, that is, $\mathbf{1}=$ $(1,0,0,0), \mathbf{i}=(0,1,0,0), \mathbf{j}=(0,0,1,0)$ and $\mathbf{k}=(0,0,0,1)$, thus elements in $\mathbb{H}$ may be expressed as follows

$$
\mathbb{H}:=\left\{\mathbf{q}=q_{1} \mathbf{1}+q_{2} \mathbf{i}+q_{3} \mathbf{j}+q_{4} \mathbf{k}, q_{1}, q_{2}, q_{3}, q_{4} \in \mathbb{R}\right\} .
$$

Quaternionic multiplication is performed in the usual manner, like polynomial multiplication, taking the following relations into account Note that the relations given in table 1 . 


\begin{tabular}{|c||c|c|c|c|}
\hline$\circ$ & $\mathbf{1}$ & $\mathbf{i}$ & $\mathbf{j}$ & $\mathbf{k}$ \\
\hline \hline $\mathbf{1}$ & $\mathbf{1}$ & $\mathbf{i}$ & $\mathbf{j}$ & $\mathbf{k}$ \\
\hline $\mathbf{i}$ & $\mathbf{i}$ & $-\mathbf{1}$ & $\mathbf{k}$ & $-\mathbf{j}$ \\
\hline $\mathbf{j}$ & $\mathbf{j}$ & $-\mathbf{k}$ & $-\mathbf{1}$ & $\mathbf{i}$ \\
\hline $\mathbf{k}$ & $\mathbf{k}$ & $\mathbf{j}$ & $-\mathbf{i}$ & $-\mathbf{1}$ \\
\hline
\end{tabular}

Table 1: Multiplication table for basis vectors

for the multiplication may be deduced directly from the ones that Hamilton gave initially, namely $\mathbf{i}^{2}=\mathbf{j}^{2}=\mathbf{k}^{2}=\mathbf{i j k}=-\mathbf{1}$.

Also we can think of $\{\mathbf{1}, \mathbf{i}, \mathbf{j}, \mathbf{k}\}$ as the four roots of unity. An alternative way of defining the quaternionic product making use of the "dot" and "cross" in $\mathbb{R}^{3}$ is given by

$$
\mathbf{q} \circ \mathbf{Q}=\left(q_{1} Q_{1}-q \cdot Q, q_{1} Q+Q_{1} q+q \times Q\right) .
$$

For the sake of a cleaner notation, we will drop the symbols $(\cdot)$ and $(\circ)$, they will be used just in case of possible confusion.

Definition (3) is, of course, directly deduced from the relations given above and may be written explicitly in term of coordinates as follows

$$
\begin{aligned}
\mathbf{q Q}= & q_{1} Q_{1}-\left(q_{2} Q_{2}+q_{3} Q_{3}+q_{4} Q_{4}\right) \\
& +\left(q_{2} Q_{1}+q_{1} Q_{2}+q_{4} Q_{3}-q_{3} Q_{4}\right) \mathbf{i} \\
& +\left(q_{3} Q_{1}-q_{4} Q_{2}+q_{1} Q_{3}+q_{2} Q_{4}\right) \mathbf{j} \\
& +\left(q_{4} Q_{1}+q_{3} Q_{2}-q_{2} Q_{3}+q_{1} Q_{4}\right) \mathbf{k} .
\end{aligned}
$$

In addition, every quaternion $\mathbf{q}=\left(q_{1}, q\right)$ has a conjugate $\overline{\mathbf{q}}=\left(q_{1},-q\right)$, that is, the real numbers are fixed by the conjugation and $\overline{\mathbf{i}}=-\mathbf{i}, \overline{\mathbf{j}}=-\mathbf{j}, \overline{\mathbf{k}}=-\mathbf{k}$. Note that $\overline{\mathbf{q} \mathbf{Q}}=\overline{\mathbf{Q}} \overline{\mathbf{q}}$.

The usual hermitian inner product is defined in $\mathbb{H}$ as

$$
\langle\mathbf{q}, \mathrm{Q}\rangle=\mathbf{q} \overline{\mathbf{Q}}
$$

such a inner product is extended in a natural way to $\mathbb{H}^{2}$, the vector space made of column vectors $(\mathbf{q}, \mathbf{Q})^{T}$, where $\mathbf{q}, \mathbf{Q} \in \mathbb{H}$

$$
\langle(\mathbf{q}, \mathbf{Q}),(\mathbf{p}, \mathbf{P})\rangle=\mathbf{q} \overline{\mathbf{Q}}+\mathbf{p} \overline{\mathbf{P}} .
$$

Note that, for the hermitian product defined above, a different choice could be made instead of (5), that is,

$$
\langle\mathbf{q}, \mathbf{Q}\rangle=\overline{\mathbf{q}} \mathbf{Q},
$$


also defines a hermitian inner product in $\mathbb{H}$.

The norm of a quaternion is denoted by $|\mathbf{q}|$, sometimes called the length of $\mathbf{q}$, it is the scalar defined by

$$
|\mathbf{q}|=\sqrt{\langle\mathbf{q}, \mathbf{q}\rangle}=\sqrt{\mathbf{q} \overline{\mathbf{q}}}=\sqrt{q_{1}^{2}+q_{2}^{2}+q_{3}^{2}+q_{4}^{2}}
$$

notice that this definition is the same that for the length of a vector in $\mathbb{R}^{4}$, or equivalently the Euclidean norm. This definition of norm is independent of the choice in the definition of the hermitian product (5) versus (7).

Finally we give the expression for the inverse, for every $\mathbf{q} \neq 0$ there exists another quaternion $\mathbf{q}^{-1}$, which will be noted as the inverse and is given by

$$
\mathbf{q}^{-1}=\frac{\overline{\mathbf{q}}}{|\mathbf{q}|^{2}}
$$

\section{Generalized Hopf map}

In this section we generalize the classical Hopf fibration from $\mathbb{C}^{2}$ to the quaternion space $\mathbb{H}^{2}$. See figure (FibracionCuaternionica).

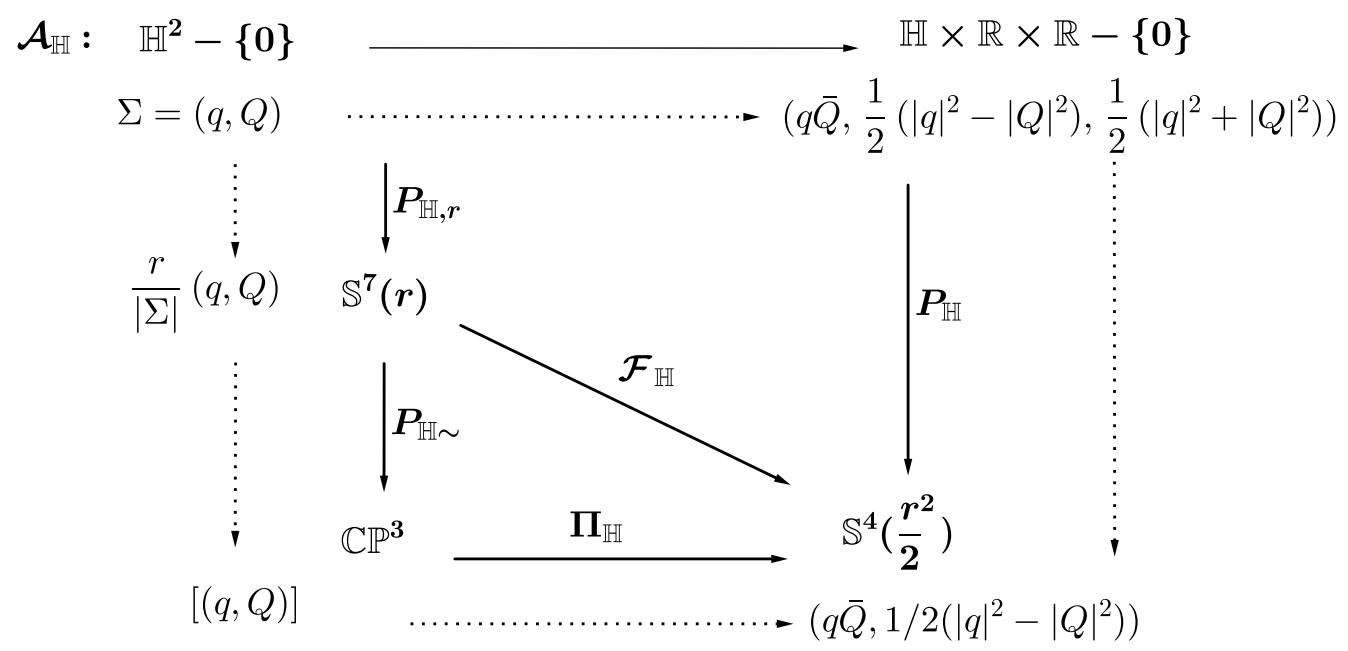

Figure 2: Generalized Hopf map and Hopf fibration.

Introducing real coordinates $\mathbf{q}=\left(q_{1}, q_{2}, q_{3}, q_{4}\right)$, through the natural equivalence $\mathbb{H} \equiv \mathbb{R}^{4}$, for the first copy of $\mathbb{H}$, and $\mathbf{Q}=\left(Q_{1}, Q_{2}, Q_{3}, Q_{4}\right)$ for the second copy of $\mathbb{H}$, we obtain a real representation of the Hopf map 


$$
\begin{aligned}
\mathcal{A}_{\mathbb{R}}(\mathbf{q}, \mathbf{Q}) & =\left(\langle\mathbf{q}, \mathbf{Q}\rangle, \frac{1}{2}\left(|\mathbf{q}|^{2}-|\mathbf{Q}|^{2}\right), \frac{1}{2}\left(|\mathbf{q}|^{2}+|\mathbf{Q}|^{2}\right)\right) \\
& =\left(\omega_{1}(\mathbf{q}, \mathbf{Q}), \omega_{2}(\mathbf{q}, \mathbf{Q}), \omega_{3}(\mathbf{q}, \mathbf{Q}), \omega_{4}(\mathbf{q}, \mathbf{Q}), \omega_{5}(\mathbf{q}, \mathbf{Q}), \omega_{6}(\mathbf{q}, \mathbf{Q})\right)
\end{aligned}
$$

where $\langle\mathbf{q}, \mathbf{Q}\rangle$ is the hermitian inner product defined by (5). Therefore, the components of $\mathcal{A}_{\mathbb{R}}$ are defined as follows

$$
\begin{aligned}
\omega_{1}(\mathbf{q}, \mathbf{Q}) & =q_{1} Q_{1}+q_{2} Q_{2}+q_{3} Q_{3}+q_{4} Q_{4}, \\
\omega_{2}(\mathbf{q}, \mathbf{Q}) & =-\left(q_{1} Q_{2}-q_{2} Q_{1}\right)-\left(q_{3} Q_{4}-q_{4} Q_{3}\right), \\
\omega_{3}(\mathbf{q}, \mathbf{Q}) & =-\left(q_{1} Q_{3}-q_{3} Q_{1}\right)+\left(q_{2} Q_{4}-q_{4} Q_{2}\right), \\
\omega_{4}(\mathbf{q}, \mathbf{Q}) & =-\left(q_{1} Q_{4}-q_{4} Q_{1}\right)-\left(q_{2} Q_{3}-q_{3} Q_{2}\right), \\
\omega_{5}(\mathbf{q}, \mathbf{Q}) & =\frac{1}{2}\left(q_{1}^{2}+q_{2}^{2}+q_{3}^{2}+q_{4}^{2}-Q_{1}^{2}-Q_{2}^{2}-Q_{3}^{2}-Q_{4}^{2}\right), \\
\omega_{6}(\mathbf{q}, \mathbf{Q}) & =\frac{1}{2}\left(q_{1}^{2}+q_{2}^{2}+q_{3}^{2}+q_{4}^{2}+Q_{1}^{2}+Q_{2}^{2}+Q_{3}^{2}+Q_{4}^{2}\right) .
\end{aligned}
$$

It is easy to check that assuming $q_{3}=q_{4}=Q_{3}=Q_{4}=0$, we get the classic Hopf map. On the other hand the Hopf variables $\omega_{i}, i=1 \ldots, 6$, satisfy

$$
\mathcal{C}\left(\omega_{1}, \omega_{2}, \omega_{3}, \omega_{4}, \omega_{5}, \omega_{6}\right)=\omega_{1}^{2}+\omega_{2}^{2}+\omega_{3}^{2}+\omega_{4}^{2}+\omega_{5}^{2}-\omega_{6}^{2}=0, \omega_{6} \geq 0,
$$

Even more, considering the algebraic manifold given by $\mathcal{C}$ and by eliminating the vertex $\mathcal{C}^{*}=\mathcal{C}-\{0\}$, it can be proven that $\mathcal{A}_{\mathbb{H}}\left(\mathbb{H}^{2}-\{0\}\right)=\mathcal{C}^{*}$. Now if we restrict $\mathcal{A}_{\mathbb{H}}$ to the 7-dimensional sphere

$$
\mathbb{S}^{7}(r)=\left\{(\mathbf{q}, \mathbf{Q}) \in \mathbb{R}^{8} \mid q_{1}^{2}+q_{2}^{2}+q_{3}^{2}+q_{4}^{2}+Q_{1}^{2}+Q_{2}^{2}+Q_{3}^{2}+Q_{4}^{2}=r^{2}=2 \omega_{6}\right\}
$$

we obtain the generalized Hopf fibration

$$
\begin{array}{cccc}
\mathcal{F}_{\mathbb{H}}(\mathbf{q}, \mathbf{Q}): & \mathbb{S}^{7}(r) & \longrightarrow & \mathbb{S}^{4}\left(\frac{r^{2}}{2}\right) \\
& (\mathbf{q}, \mathbf{Q}) & \rightsquigarrow & \left(\omega_{1}, \omega_{2}, \omega_{3}, \omega_{4}, \omega_{5}\right)
\end{array}
$$

where $\mathbb{S}^{4}\left(\frac{r^{2}}{2}\right)$ is the 4 -dimensional sphere.

A different choice of the hermitian inner product in the definition of $\mathcal{A}_{\mathbb{R}}$, as defined by (7), leads to an analogous scheme. Under this condition the components $\omega_{1}, \omega_{5}, \omega_{6}$ of $\mathcal{A}_{\mathbb{R}}$ remain the same and $\omega_{2}, \omega_{3}, \omega_{4}$ become the following three new functions

$$
\begin{aligned}
& G_{1}(q, Q)=\left(\left(q_{1} Q_{2}-q_{2} Q_{1}\right)-\left(q_{3} Q_{4}-q_{4} Q_{3}\right)\right), \\
& G_{2}(q, Q)=\left(\left(q_{1} Q_{3}-q_{3} Q_{1}\right)+\left(q_{2} Q_{4}-q_{4} Q_{2}\right)\right) \\
& G_{3}(q, Q)=\left(\left(q_{1} Q_{4}-q_{4} Q_{1}\right)-\left(q_{2} Q_{3}-q_{3} Q_{4}\right)\right) .
\end{aligned}
$$


The diagram given in Figure (2) keeps its validity with the slight changes on the definition of $\mathcal{A}_{\mathbb{R}}$ given above, even more, relation (10) is transformed into

$$
\mathcal{C}\left(\omega_{1}, G_{1}, G_{2}, G_{3}, \omega_{5}, \omega_{6}\right)=\omega_{1}^{2}+G_{1}^{2}+G_{2}^{2}+G_{3}^{2}+\omega_{5}^{2}-\omega_{6}^{2}=0, \omega_{6} \geq 0 .
$$

\section{Reduction of the 4DOF harmonic oscillator}

Consider on $\mathbb{R}^{8}$, with co-ordinates $(q, Q)=\left(q_{1}, q_{2}, q_{3}, q_{4}, Q_{1}, Q_{2}, Q_{3}, Q_{4}\right)$, and standard symplectic form $\omega$, the isotropic harmonic oscillator with Hamiltonian

$$
H_{2}(q, Q)=\frac{1}{2}\|q\|^{2}+\frac{1}{2}\|Q\|^{2}
$$

To get a convenient ordering we will rename the functions obtained so far. Let

$$
\sigma_{7}(q, Q)=G_{1}(q, Q), \quad \sigma_{8}(q, Q)=G_{2}(q, Q), \quad \sigma_{9}(q, Q)=G_{3}(q, Q)
$$

The Hamiltonian flows corresponding to $\sigma_{7}, \sigma_{8}, \sigma_{9}$ generate a group $G$, which is a symmetry group for the system $\left(H_{2}, \omega, \mathbb{R}^{8}\right)$, because $\left\{\sigma_{7}, H_{2}\right\}=\left\{\sigma_{8}, H_{2}\right\}=\left\{\sigma_{9}, H_{2}\right\}=0$, where $\{$,$\} is the Poisson bracket associated to the standard symplectic form \omega$. Furthermore $\left\{G_{i}, G_{j}\right\}=\varepsilon_{i j k} G_{k}$, thus these functions form a basis for the Lie algebra $\mathfrak{s o}(3)$ and $G=S O(3)$.

Next consider the functions

$$
\begin{aligned}
& \sigma_{1}(q, Q)=\omega_{2}(q, Q)=-\left(\left(q_{1} Q_{2}-q_{2} Q_{1}\right)+\left(q_{3} Q_{4}-q_{4} Q_{3}\right)\right), \\
& \sigma_{2}(q, Q)=\omega_{3}(q, Q)=-\left(\left(q_{1} Q_{3}-q_{3} Q_{1}\right)-\left(q_{2} Q_{4}-q_{4} Q_{2}\right)\right), \\
& \sigma_{3}(q, Q)=\omega_{4}(q, Q)=-\left(\left(q_{1} Q_{4}-q_{4} Q_{1}\right)+\left(q_{2} Q_{3}-q_{3} Q_{2}\right)\right), \\
& \sigma_{4}(q, Q)=\omega_{1}(q, Q)=<q, Q> \\
& \sigma_{5}(q, Q)=\omega_{5}(q, Q)=\frac{1}{2}\|q\|^{2}-\frac{1}{2}\|Q\|^{2}, \\
& \sigma_{6}(q, Q)=\omega_{6}(q, Q)=\frac{1}{2}\|q\|^{2}+\frac{1}{2}\|Q\|^{2} .
\end{aligned}
$$

Note that these same polynomials also play a role in the reduction of the Kepler system [4] and the reduction of the 4DOF isotropic harmonic oscillator [5, 6, 7].

The polynomials $\sigma_{1}, \sigma_{2}, \sigma_{3}$ span a Lie algebra isomorphic to $\mathfrak{s o}(3)$. Furthermore $\left\{\sigma_{4}, \sigma_{5}\right\}=$ $-2 \sigma_{6},\left\{\sigma_{4}, \sigma_{6}\right\}=-2 \sigma_{5},\left\{\sigma_{5}, \sigma_{6}\right\}=2 \sigma_{4}$, that is, $\sigma_{4}, \sigma_{5}, \sigma_{6}$ span a Lie algebra isomorphic to $\mathfrak{s u}(1,1) \cong \mathfrak{s l}(2, \mathbb{R})$. In addition $\sigma_{7}, \sigma_{8}, \sigma_{9}, \sigma_{1}, \sigma_{2}, \sigma_{3}$ span a Lie algebra isomorphic to $\mathfrak{s o}(3) \times \mathfrak{s o}(3) \cong \mathfrak{s o}(4)$ and $\left\{\sigma_{i+6}, \sigma_{j}\right\}=\left\{\sigma_{i}, \sigma_{j}\right\}=0$ for $i=1,2,3$, and $j=4,5,6$.

Note that $\mathfrak{s l}(2, \mathbb{R})$ generated by $\sigma_{4}, \sigma_{6}+\sigma_{5}, \sigma_{6}-\sigma_{5}$ gives the full linear Lie algebra invariant under the $S O(4)$ action generated by $\sigma_{7}, \sigma_{8}, \sigma_{9}, \sigma_{1}, \sigma_{2}, \sigma_{3}$ (see [12]). If we 
consider $\mathfrak{s o}(4) \cong \mathfrak{s o}(3) \times \mathfrak{s o}(3)$, with the first copy of $\mathfrak{s o}(3)$ generated by $\sigma_{7}, \sigma_{8}, \sigma_{9}$ and the second copy of $\mathfrak{s o}(3)$ generated by $\sigma_{1}, \sigma_{2}, \sigma_{3}$ then the full linear Lie algebra of invariants for the first copy of $\mathfrak{s o}(3)$ is $\mathfrak{s o}(3) \times \mathfrak{s l}(2, \mathbb{R})$ generated by $\sigma_{1}, \sigma_{2}, \sigma_{3}, \sigma_{4}, \sigma_{5}, \sigma_{6}$, and the full linear Lie algebra of invariants for the second copy of $\mathfrak{s o}(3)$ is $\mathfrak{s o}(3) \times \mathfrak{s l}(2, \mathbb{R})$ generated by $\sigma_{7}, \sigma_{8}, \sigma_{9}, \sigma_{4}, \sigma_{5}, \sigma_{6}$. Thus polynomials $\sigma_{i}$ are invariants for the $G$-action and are generators for the space of $G$-invariant polynomials. Consequently the orbit map for the $G$-action is

$$
\rho: \mathbb{R}^{8} \rightarrow \mathbb{R}^{6} ;(q, Q) \rightarrow\left(\sigma_{1}, \cdots, \sigma_{6}\right) .
$$

Consequently, $\rho$ is equivalent to the real representation of the Hopf map given in (8).

Let $S_{i j}(q, Q)=q_{i} Q_{j}-q_{j} Q_{i}$ Then

$$
\sum_{1 \leqslant i<j \leqslant 4} S_{i j}^{2}=\|q\|^{2}\|Q\|^{2}-<q, Q>^{2}=-\sigma_{5}^{2}+\sigma_{6}^{2}-\sigma_{4}^{2}
$$

and

$$
S_{12} S_{34}-S_{13} S_{24}+S_{14} S_{23}=0
$$

Thus

$$
\sigma_{1}^{2}+\sigma_{2}^{2}+\sigma_{3}^{2}=-\sigma_{5}^{2}+\sigma_{6}^{2}-\sigma_{4}^{2},
$$

and

$$
\sigma_{7}^{2}+\sigma_{8}^{2}+\sigma_{9}^{2}=-\sigma_{5}^{2}+\sigma_{6}^{2}-\sigma_{4}^{2} .
$$

Thus the orbit space $\rho\left(\mathbb{R}^{8}\right)$ for the above defined $S O(3)$ representation is five dimensional and is determined by (17). The reduced phase spaces for the $G$-action on the one hand are the co-adjoint orbits for the action of $S O(3)$ on $\mathfrak{s o}(3) \times \mathfrak{s o}(3)$, and on the other hand are determined by the $\mathfrak{s o}(3)$ Casimir $C(q, Q)=\sigma_{7}^{2}+\sigma_{8}^{2}+\sigma_{9}^{2}$. Therefore the reduced phase spaces are $\rho\left(C^{-1}(c)\right)$, i.e. the subset of $\mathbb{R}^{6}$ determined by

$$
\begin{aligned}
\sigma_{1}^{2}+\sigma_{2}^{2}+\sigma_{3}^{2} & =c, \\
-\sigma_{5}^{2}+\sigma_{6}^{2}-\sigma_{4}^{2} & =c .
\end{aligned}
$$

Thus, if $c \neq 0$, the reduced phase space is the four dimensional product of a two-sphere and a two-sheeted hyperboloid. If $\mathrm{c}=0$ we have a critical two dimensional reduced phase space which is a cone times a point.

When we consider the harmonic oscillator Hamiltonian then $H_{2}^{-1}(h)$ is $\mathbb{S}^{7} \subset \mathbb{R}^{8}$ and

$$
\rho\left(H_{2}^{-1}(h)\right): \mathbb{S}^{7} \rightarrow \mathbb{S}^{4}
$$

is a generalized Hopf map giving the generalized Hopf fibration of the seven-sphere (see $[2])$. The $\mathbb{S}^{4}$ is given by

$$
\sigma_{1}^{2}+\sigma_{2}^{2}+\sigma_{3}^{2}+\sigma_{4}^{2}+\sigma_{5}^{2}=h^{2} .
$$


This four-sphere intersects the $S O(3)$-reduced phase spaces $\rho\left(C^{-1}(c)\right)$ in the sets

$$
\begin{aligned}
& \sigma_{1}^{2}+\sigma_{2}^{2}+\sigma_{3}^{2}=c, \\
& \sigma_{4}^{2}+\sigma_{5}^{2}=h^{2}-c, h \leqslant c \leqslant 0,
\end{aligned}
$$

which are topologically $\mathbb{S}^{2} \times \mathbb{S}^{1}$. The sets given by (20) are not reduced phase spaces for a combined reduction with respect to the $S O(3)$ action by $G$ and the $\mathbb{S}^{1}$ action generated by $H_{2}$, because the induced $H_{2}$-action on $\rho\left(C^{-1}(c)\right) \cap \rho\left(H_{2}^{-1}(h)\right.$ is an $\mathbb{S}^{1}$ action which is trivial on $\sigma_{1}^{2}+\sigma_{2}^{2}+\sigma_{3}^{2}=c$ and which rotates along $\sigma_{4}^{2}+\sigma_{5}^{2}=h-c$. An additional reduction with respect to the $\mathrm{H}_{2}$-action will therefore result in a two dimensional reduced phase space defined by $\sigma_{1}^{2}+\sigma_{2}^{2}+\sigma_{3}^{2}=c$.

One should be aware that the above construction depends on the choices of the real coordinates for the quaternion representation. An other choice will alter the way the Lie algebra structure of the quaternions is represented and change the role of the harmonic oscillator Hamiltonian with respect to the Poisson bracket induced by the standard symplectic form.

\section{The twistor fibration}

In the above we have shown that a generalized Hopf map is present in the dynamics of the isotropic harmonic oscillator on $\mathbb{R}^{8}$ by explicit construction. Actually we constructed some representation of the map $\mathcal{A}_{\mathbb{H}} \circ P_{\mathbb{H}}$ in the diagram in figure (2), which is a restriction of an orbit map for a symplectic group action. We know that the map $\Pi_{\mathbb{H}}$ in this diagram represents the twistor fibration (see [1]) or the Calabi-Hopf-Penrose fibration $\mathbb{C P}^{1} \hookrightarrow$ $\mathbb{C P}^{3} \rightarrow S^{4}$ which is obtained via a quotient of two Hopf maps [8]:

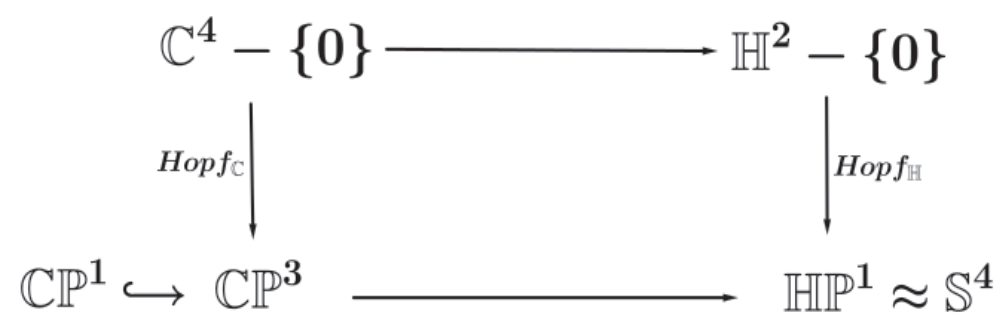

Figure 3: Twistor fibration

In this section we will show how the twistor fibration can be obtained by factorizing the orbit map for the symplectic $S O(3)$-action through the orbit map for a symplectic $S O(2)$-action.

One should notice that the linear Lie algebra of $S O(3)$ invariants will be noncompact due to the presence of the $\mathfrak{s l}(2, \mathbb{R})$ subalgebra. Because the linear Lie algebra of invariants for 
the $H_{2}$ action is isomorphic to the compact Lie algebra $\mathfrak{s u}(4)$, it is impossible to perform the above constructed $S O(3)$ reduction in a way that factors through the $\mathrm{H}_{2}$ reduction.

If in the diagram in figure(2) the map $P_{\mathbb{H}, r} \circ P_{\mathbb{H}, \sim}$ would describe an $H_{2}$ reduction, than this would imply an $S O(3)$ reduction factoring through the $H_{2}$ reduction. Consequently the second map $P_{\mathbb{H}, \sim}$ is not an $H_{2}$-reduction, although it is known that the reduced phase space for the $H_{2}$-action is $\mathbb{C P}^{3}$ (see [10]).

Choose $\sigma_{7}$ from the representation of the $S O(3)$ group action. Then $\left\{\sigma_{7}, H_{2}\right\}=0$ and we can identify $\mathbb{H}^{2}$ with $\mathbb{C}^{4}$ in such a way that the action of $\sigma_{7}$ is given by $e^{i t} z, z \in \mathbb{C}^{4}$. Let $\rho_{\sigma_{7}}$ be the orbit map for the flow of $\sigma_{7}$. Then $\rho_{\sigma_{7}}\left(H_{2}^{-1}(h)\right) \cong \mathbb{C P}^{3}$. More details on the action of $\sigma_{7}$ can be found in [9]. Consequently the orbit map for the symplectic $\sigma_{7}$-action, when restricted to $H_{2}(q, Q)=h$, coincides with the map Hopf $\mathbb{C}_{\mathbb{C}}$ in the diagram given in figure (3) and with $P_{\mathbb{H}, r} \circ P_{\mathbb{H}, \sim}$ in figure(2). Now the $\sigma_{7}$-action is a subgroup of the $S O(3)$-action giving $S O(3) / S O(2) \cong S^{2} \cong S^{3} / S^{1} \cong \mathbb{C P}^{1}$ as the fiber of the twistor fibration, where $S^{3} / S^{1}$ is the Hopf fibration. It is now clear that the map $\Pi_{\mathbb{H}}$ in figure(2) cannot be an orbit map for a symplectic group action, i.e. $S O(3)$ reduction cannot be performed by symplectic reduction in stages.

\section{Acknowledgements}

Support from the Government of Spain is acknowledged. It came in the form of research projects MTM 2012-31883, of the Ministry of Science and 12006/PI/09 from Fundación Séneca of the Autonomous Region of Murcia.

\section{References}

[1] Baird, P. : An Introduction to Twistors, http://www.math.jussieu.fr/ helein/encyclopaedia/baird-twistors.pdf

[2] Crespo, F., and Ferrer, S.: Classical flows among a large parametric family $\mathcal{F}_{a}$., In preparation, Universidad de Murcia, 2012.

[3] Cushman, R.H. and Rod, D.L.: Reduction of the semisimple 1:1 resonance, Physica D, 6, pp 105-112, 1982 .

[4] Cushman, R.H. and Bates, L.M.: Global Aspects of Classical Integrable Systems; Birkhäuser Verlag, Basel, 1997.

[5] Diaz, G., Egea, J., Ferrer, S., Meer, J.C. van der, Vera, J.A.: Relative equilibria and bifurcations in the generalized Van der Waals 4-D oscillator. Physica D, 239, pp 1610-1625, 2010. 
[6] Egea, J., Ferrer, S., and van der Meer, J.C.: Hamiltonian Fourfold 1:1 Resonance with Two Rotational Symmetries, Regular and Chaotic Dynamics 12, pp 664 -674, 2007.

[7] Egea, J., Ferrer, S., and Van der Meer, J.C. : Bifurcations of the Hamiltonian fourfold 1:1 resonance with toroidal symmetry, Journal of Nonlinear Science, 21, pp 835-874, 2011.

[8] Loo, B, F., and Verjovsky, A.: The Hopf fibration over $S^{8}$ admits no $S^{1}$-subfibration, Topology 31, pp 239-254, 1992.

[9] van der Meer, J.C. : The Kepler system as a reduced 4D harmonic oscillator, CASA report 13-23, Technische Universiteit Eindhoven, 2013.

[10] Moser, J. : Regularization of Kepler's Problem and the Averaging Method on a Manifold ; Comm. Pure Appl. Math. 23, pp 609-636, 1970.

[11] Hamilton, W.R.: On Quaternions; or a new system of imaginaries in algebra ; Philos. Mag. 25, pp 489-495, 1844.

[12] Weyl, H: The Clasical Groups. Their Invariants and Representations. Princeton Univ. Press, Princeton, New Jersey, 1939. 
PREVIOUS PUBLICATIONS IN THIS SERIES:

\begin{tabular}{|c|c|c|c|}
\hline Number & Author(s) & Title & Month \\
\hline 14-09 & $\begin{array}{l}\text { O. Krehel } \\
\text { A. Muntean } \\
\text { T. Aiki }\end{array}$ & $\begin{array}{l}\text { A thermo-diffusion system } \\
\text { with Smoluchowski } \\
\text { interactions: well-posedness } \\
\text { and homogenization }\end{array}$ & Apr. 'I4 \\
\hline I4-IO & $\begin{array}{l}\text { N. Banagaaya } \\
\text { G. Ali } \\
\text { W.H.A. Schilders }\end{array}$ & $\begin{array}{l}\text { Implicit-IMOR method for } \\
\text { index-I and index-2 linear } \\
\text { constant DAEs }\end{array}$ & Apr. 'I4 \\
\hline I4-II & $\begin{array}{l}\text { M.H. Duong } \\
\text { M. Fathi }\end{array}$ & $\begin{array}{l}\text { The two-scale approach to } \\
\text { hydrodynamic limits for } \\
\text { non-reversible dynamics }\end{array}$ & Apr. 'I4 \\
\hline I4-I 2 & $\begin{array}{l}\text { O. Krehel } \\
\text { A. Muntean } \\
\text { P. Knabner }\end{array}$ & $\begin{array}{l}\text { Multiscale modeling of } \\
\text { colloidal dynamics in } \\
\text { porous media: Capturing } \\
\text { aggregation and deposition } \\
\text { effects }\end{array}$ & Apr. 'I4 \\
\hline I4-I3 & $\begin{array}{l}\text { J.C. van der Meer } \\
\text { F. Crespo } \\
\text { S. Ferrer }\end{array}$ & $\begin{array}{l}\text { Generalized Hopf fibration } \\
\text { and geometric } \mathrm{SO}(3) \\
\text { reduction of the } 4 \mathrm{DOF} \\
\text { harmonic oscillator }\end{array}$ & Apr. 'I4 \\
\hline
\end{tabular}

